occurred frequently because of excessive tension, deficiency of blood flow at the site of anastomosis, or both. ${ }^{5}$ We successfully performed right upper lobectomy of the lung using extended wedge resection of the tracheal carina, and this technique was as easy and safe as the tracheobronchoplastic procedure. Furthermore, because of the nature of the tumor without diffuse infiltration, tumor-free surgical margins were obtained in this case.

This is the first case report of primary pulmonary malignant schwannoma involving the tracheal carina, which was successfully removed by using the extended bronchoplastic procedure.

\section{References}

1. Togashi K, Hirahara H, Sugawara M, Oguma F. Primary malignant schwannoma of the lung. Jpn J Thorac Cardiovasc Surg. 2003;51:692-5.

2. Wong WW, Hirose T, Scheithauer BW, et al. Malignant peripheral nerve sheath tumor: analysis of treatment outcome. Int $J$ Radiat Oncol Biol Phys. 1998;42:351-60.

3. Janssen JP, Mulder JJ, Wagenaar SS, et al. Primary sarcoma of the lung: a clinical study with long-term follow-up. Ann Thorac Surg. 1994;58:1151-5.

4. Keel SB, Bacha E, Mark EJ, et al. Primary pulmonary sarcoma: a clinicopathologic study of 26 cases. Mod Pathol. 1999;12:1124-31.

5. Grillo HC. Carinal reconstruction. Ann Thorac Surg. 1982;34:356-73.

\title{
Video-assisted mediastinoscopy improved radical resection for cancer in transhiatal esophagectomy
}

\author{
Daniel Pop, MD, Nicolas Venissac, MD, and Jerome Mouroux, MD, Nice, France
}

T ranshiatal esophagectomy (THE), popularized by Orringer and Sloan, ${ }^{1}$ was proposed to decrease postoperative morbidity and mortality. The major inconvenience is the mediastinal lymphadenectomy that guarantees radical oncologic surgery. Recently, Bumm and associates $^{2}$ used an endodissector that eliminated the "blind" mediastinal dissection. Furthermore, the advent of video-assisted technology provided increasing visualization and allowed bimanual maneuvers. ${ }^{3,4}$ This is our preliminary report using videoassisted mediastinoscopy (VAM) during THE, including technical details.

\section{Patients and Techniques}

Between October 1, 2001 and January 31, 2003 (a 15-month period), we operated on our first 5 patients. The usual pretherapeutic staging and preoperative assessment were done. One patient had neoadjuvant chemoradiotherapy. All 5 patients' characteristics are noted in Table 1 .

The surgical procedures were done by the same team (surgeon with two assistants). The patients were supine with one roll beneath the scapulae (for maximal cervical extension). The abdominal and cervical phases were done by the standard technique. ${ }^{1}$ Care must be taken to avoid arrhythmia or hypotension because of cardiac displacement during abdominal upward dissection of the esophagus. The mediastinal phase is done

From the Thoracic Surgery Department, Pasteur Hospital, Nice, France.

Received for publication June 24, 2006; revisions received Aug 10, 2006; accepted for publication Aug 25, 2006.

Address for reprints: Daniel Pop, MD, Thoracic Surgery Department, Pasteur Hospital-Building H1, 30 Avenue de la Voie Romaine, 06002 Nice, France (E-mail: danielpopch@yahoo.com).

J Thorac Cardiovasc Surg 2007;133:267-8

$0022-5223 / \$ 32.00$

Copyright $\odot 2007$ by The American Association for Thoracic Surgery doi:10.1016/j.jtcvs.2006.08.066 by VAM only through a cervicotomy (Figure 1). The equipment and the instruments have been previously described. ${ }^{4}$ First, along the left side of the esophagus, the video-mediastinoscope allows clear visualization of the left recurrent nerve to the aortopulmonary window level. With bimanual blunt dissection and hemostatic clips, the lymph nodes situated next to the nerve can be easily separated without hurting the nerve. The video-mediastinoscope can be pushed farther into the mediastinum until the full length is reached. Next the anterior side is dissected. The back surface of the trachea, the carina, and the lymph nodes can be safely detached. Then the posterior side of the esophagus can be easily dissected from the prevertebral fascia. The thoracic duct can be identified and endoclips can be used generously for lymphostasis. Finally, the right side of the esophagus can be dissected by passing behind or in front of the esophagus, avoiding injury to the azygos system. Normally, the mediastinal pleurae are not opened. When the superior mediastinal phase is accomplished, the video-mediastinoscope provides the light to reach the upward abdominal dissection of the esophagus. We prefer a gastric substitute. One patient needed a left colon graft because of total gastrectomy for synchronous antral cancer, which lengthened the intervention. The mean operative time for the remainder was about 280 minutes. The mean

TABLE 1. Patients' characteristics

\begin{tabular}{lccccc}
\hline $\begin{array}{l}\text { No. (age [y], } \\
\text { sex) }\end{array}$ & $\begin{array}{c}\text { IT/length } \\
\text { (cm) }\end{array}$ & \multicolumn{1}{c}{ uTN/pTN } & FEV $_{\mathbf{1}}(\mathbf{L} / \%)$ & $\begin{array}{c}\text { Surgery } \\
(\mathbf{m i n})\end{array}$ & $\begin{array}{c}\text { Survival } \\
(\mathbf{m o})\end{array}$ \\
\hline $1(73, \mathrm{~F})$ & $29 / 2$ & T1 N1/T1 N0 & $2.2(130)$ & 220 & $42-\mathrm{AWD}$ \\
$2(70, \mathrm{M})$ & $25 / 5$ & T3 N0/T3 N0 & $1.5(54)$ & 330 & $37-\mathrm{AWD}$ \\
$3(51, \mathrm{M})$ & $27 / 6$ & T3 N1/T3 N1 & $2.7(91)$ & 480 & $17-\mathrm{DWD}$ \\
$4(76, \mathrm{~F})$ & $31 / 2$ & T2 N1/T2 N1 & $0.6(33)$ & 255 & $51-\mathrm{AWD}$ \\
$5(63, \mathrm{M})$ & $32 / 7$ & Barrett/Tis & $3.2(106)$ & 325 & $36-$ AWD \\
\hline
\end{tabular}

$I T$, Distance from incisors in flexible esophagoscopy; $u$, endoscopic ultrasound; $p$, postoperative; Barrett, Barrett esophagus; Tis, carcinoma in situ; $\mathrm{FEV}_{1}$, forced expiratory volume in 1 second (liter and \%); $A W D$, alive without disease; $D W D$, dead without disease. 


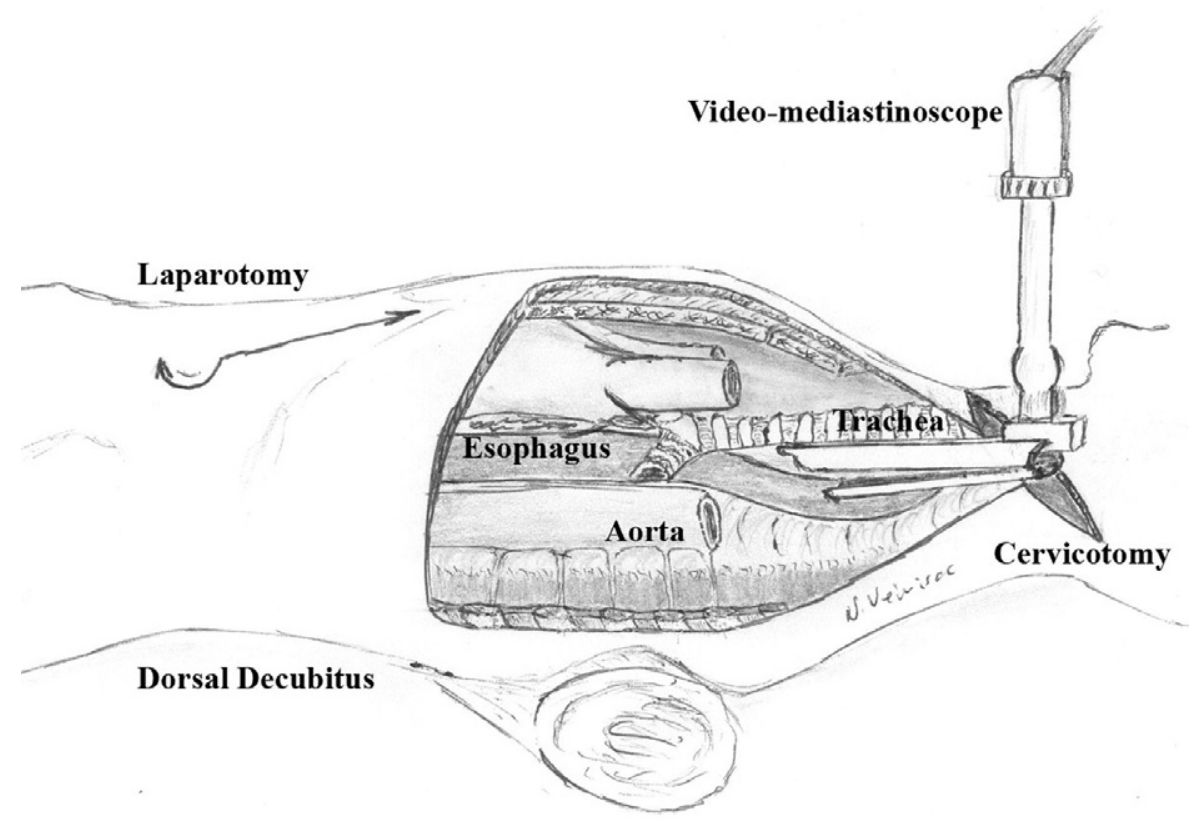

Figure 1. Mediastinal phase of THE: The mediastinoscope is inserted in the inferior pole of the standard cervicotomy. The VAM dissection is done around the esophagus behind the trachea.

hospital stay was 21 days. There was no 30-day mortality. Two major complications arose: myocardial ischemia in a patient with known coronary stenosis and pneumonia in a patient with acute respiratory distress syndrome that required ventilatory support. The living patients had no recurrences; one patient died of a myocardial infarction, but was free of cancer.

\section{Discussion}

Use of a vein stripper to avulse the esophagus from the posterior mediastinum was first described by Denk (1913) in cadavers and experimental animals. ${ }^{1}$ After several attempts by different teams, Orringer and Sloan ${ }^{1}$ (1978) reported on 26 patients with THE with gastric or colonic replacement in the same operative stage. The anticipated benefit was to avoid the morbidity of thoracotomy and to lessen the disastrous effect of an anastomotic fistula. Critics of this approach have warned of inadequate hemostasis and oncologic surgery.

Recently, a meta-analysis ${ }^{5}$ showed that perioperative blood loss was significantly higher after transthoracic esophagectomy (TTE) but that THE resulted in more severe bleeding. The postoperative outlook gave potential benefits for THE in terms of pulmonary complications, chylous leakage, and in-hospital mortality. To the contrary, THE resulted in more cardiac complications, vocal cord paralysis, and anastomotic leakage. The use of mediastinoscopy ${ }^{2}$ helps dissection at or above the trachea and reduces the postoperative complications of standard THE. The advent of the video camera substantially improves visualization. $\mathrm{We}^{4}$ have proved the benefit in resecting paratracheal mesothelial cysts. None of our patients treated by THE had significant bleeding or recurrent nerve injury.
The primary goal of cancer surgery remains radical resection. Partial esophagectomy and 2-field lymphadenectomy done by TTE is the most currently used technique. Theoretically, it offers a better, but not significantly better, 5 -year survival. ${ }^{5}$ The new VAM technique and the possibility of bimanual handling allow true lymphadenectomy. ${ }^{3}$ For our patients, the mean number of lymph nodes was 7 (limits 2-22). This technique enables a truly en bloc esophagectomy and lymphadenectomy.

Despite our limited experience with THE and VAM, the results are promising. With careful handling, VAM is superior to standard mediastinoscopy and it reduces the complications linked to the surgical maneuvers (inadequate hemostasis and recurrent nerve injury). In our opinion, VAM allows not only controlled lymph node biopsy in the mediastinum but true lymphadenectomy around the esophagus.

\section{References}

1. Orringer MB, Sloan H. Esophagectomy without thoracotomy. J Thorac Cardiovasc Surg. 1978;76:643-54.

2. Bumm R, Hölscher AH, Feussner H, Tachibana M, Bartels H, Siewert JR. Endodissection of the thoracic esophagus: technique and clinical results in transhiatal esophagectomy. Ann Surg. 1993;218:97-104.

3. Hürtgen M, Friedel G, Toomes H, Fritz P. Radical video-assisted mediastinoscopic lymphadenectomy (VAMLA) - technique and first results. Eur J Cardiothorac Surg. 2002;21:348-51.

4. Pop D, Venissac N, Leo F, Mouroux J. Video-assisted mediastinoscopy: a useful technique for paratracheal mesothelial cysts. J Thorac Cardiovasc Surg. 2005;129:690-1.

5. Hulscher JBF, Tijssen JGP, Obertop H, van Lanschot JJB. Transthoracic versus transhiatal resection for carcinoma of the esophagus: a meta-analysis. Ann Thorac Surg. 2001;72:306-13. 